\title{
Silencing the buzz: a new approach to population suppression of mosquitoes by feeding larvae double-stranded RNAs
}

Steve Whyard ${ }^{1 *}$, Cassidy NG Erdelyan ${ }^{1}$, Alison L Partridge ${ }^{1}$, Aditi D Singh ${ }^{1}$, Nigel W Beebe ${ }^{2,3}$ and Rupert Capina ${ }^{1}$

\begin{abstract}
Background: Mosquito-borne diseases threaten over half the world's human population, making the need for environmentally-safe mosquito population control tools critical. The sterile insect technique (SIT) is a biological control method that can reduce pest insect populations by releasing a large number of sterile males to compete with wild males for female mates to reduce the number of progeny produced. Typically, males are sterilized using radiation, but such methods can reduce their mating competitiveness. The method is also most effective if only males are produced, but this requires the development of effective sex-sorting methods. Recent efforts to use transgenic methods to produce sterile male mosquitoes have increased interest in using SIT to control some of our most serious disease vectors, but the release of genetically modified mosquitoes will undoubtedly encounter considerable delays as regulatory agencies deal with safety issues and public concerns.
\end{abstract}

Methods: Testis genes in the dengue vector Aedes aegypti were identified using a suppression subtractive hybridization technique. Mosquito larvae were fed double-stranded RNAs (dsRNAs) that targeted both the testis genes and a female sex determination gene (doublesex) to induce RNA interference (RNAi) -mediated sterility and inhibition of female development. Fertility and mating competiveness of the treated males were assessed in small-scale mating competition experiments.

Results: Feeding mosquito larvae dsRNAs targeting testis genes produced adult males with greatly reduced fertility; several dsRNAs produced males that were highly effective in competing for mates. RNAi-mediated knockdown of the female-specific isoform of doublesex was also effective in producing a highly male-biased population of mosquitoes, thereby overcoming the need to sex-sort insects before release.

Conclusions: The sequence-specific gene-silencing mechanism of this RNAi technology renders it adaptable for species-specific application across numerous insect species. We envisage its use for traditional large-scale reared releases of mosquitoes and other pest insects, although the technology might also have potential for field-based control of mosquitoes where eggs deposited into a spiked larval site lead to the release of new sterile males.

Keywords: Sterile insect technique, RNA interference, Testis, Spermatogenesis, Aedes aegypti, Dengue, Doublesex

\footnotetext{
* Correspondence: Steve.Whyard@umanitoba.ca

'Department of Biological Sciences, University of Manitoba, Winnipeg, MB,

Canada

Full list of author information is available at the end of the article
}

\section{Biomed Central}

(c) 2015 Whyard et al.; licensee BioMed Central. This is an Open Access article distributed under the terms of the Creative Commons Attribution License (http://creativecommons.org/licenses/by/4.0), which permits unrestricted use, distribution, and reproduction in any medium, provided the original work is properly credited. The Creative Commons Public Domain Dedication waiver (http://creativecommons.org/publicdomain/zero/1.0/) applies to the data made available in this article, unless otherwise stated. 


\section{Background}

Mosquitoes are the world's most serious disease vectors, transmitting parasites and viruses that infect hundreds of millions annually. Given the deficit of effective vaccines for these diseases, our best defence has been mosquito control [1]. Broad-spectrum insecticides have been used for decades to control mosquitoes, but growing concerns about the adverse effects of these chemicals on other beneficial species, and increasing incidences of insecticide resistance amongst the vectors themselves, have prompted renewed interest in species-specific control methods [1]. The sterile insect technique (SIT) is a non-insecticidal control method that relies on the release of sterile male mosquitoes that search for and mate with wild females, preventing offspring. This approach has been used successfully to control various insect pest species [2], including the New World screwworm fly [3], the tsetse fly [4], the Mediterranean fruit fly [5], and the apple codling moth [6]. Radiation or chemosterilants are often used to sterilize males, but such treatments can decrease the insects' ability to compete with wild males for mates, often necessitating the production of more sterile males to increase efficacy $[7,8]$. SIT is also generally more effective if females are not released [9], as sterile female insects can still damage crops, transmit disease, or simply distract sterile males from finding wild mates, which adds the complexity of sex-sorting to the process of an effective SIT approach.

The first applications of SIT to mosquitoes encountered mixed successes during the 1960s, 70s, and 80s [10], as it proved difficult to produce sufficient numbers of competitive sterile males to suppress natural populations. More recently, elegant transgenic approaches have generated sterile male mosquitoes for the malaria vector Anopheles gambiae [11,12], the Asian tiger mosquito Aedes albopictus [13], and the dengue vector Aedes aegypti $[14,15]$. Preliminary releases of transgenic sterile Ae. aegypti in Grand Cayman have demonstrated an effective reduction of these mosquitoes [16]. But while transgenic approaches have renewed interest in SIT for mosquito control, the use of genetically-modified (GM) insects will require continued effort at the technical level to develop field-competitive strains adapted for different locations, and at the regulatory level to address public concerns about the possible impacts of GM insects $[17,18]$, particularly as mosquito movements are not restricted by borders.

Given that some countries will require considerable time to resolve these regulatory and public concerns about GM technologies, we began to develop an alternative, non-GM method that delivered both mosquito sterilization and sex sorting using RNAi technologies. RNAi is a gene-silencing mechanism achieved by delivering double-stranded RNA (dsRNA) to cells or organisms [19]. As it was already known that dsRNA could be fed to mosquitoes to induce detectable RNAi [20,21], we hypothesized that RNAi could be used to produce effective sterile male Ae. aegypti mosquitoes by targeting genes associated with mosquito male testes and female sex determination genes.

\section{Methods}

Subtractive library construction and differential screening

Testes and ovaries were dissected from 2-day old adult $\mathrm{Ae-}$ des aegypti mosquitoes. mRNA was isolated from these tissues, the rest of the bodies (minus gonads), and fourth instar larvae using an Oligotex mRNA Mini Kit (Qiagen). Suppression subtractive hybridization ( $\mathrm{SSH}$ ) was used to identify genes that were preferentially expressed in Ae. aegypti testes relative to other tissues within the male mosquito's body. The testis-specific subtracted library was built using a PCR-Select cDNA Subtraction kit (Clontech), using testis cDNA as the TESTER source of cDNA, and using cDNA derived from the rest of the body as the DRIVER cDNA. The SSH-specific adapters were ligated to the TESTER cDNAs and the two pools of cDNA were hybridized for the forward subtracted library. Reverse subtracted libraries were built for subsequent differential screening, where the TESTER and DRIVER designations were inversed. Amplification of hybrids corresponding to common sequences was suppressed, yielding a library enriched for differentially expressed sequences within the testes. The forward-subtracted library was ligated into the pDrive plasmid vector (Qiagen) and used to transform DH5 $\alpha$ E. coli cells (Invitrogen).

The efficiency of the subtraction of the testes library was estimated using qRT-PCR by comparing the abundance of a predicted non-differentially expressed gene, $\beta$-tubulin, using the primers tubF (CGTCGTAGAACCGTACAAC) and tubR (CAGGCAGGTGGTAATCC). The testissubtracted library was screened for differentially expressed ESTs using a PCR-select cDNA subtraction screening kit (Clontech). Briefly, 150 Escherichia coli clones were selected randomly for screening, plated, and subjected to duplicate bacterial colony lifts using standard techniques. The filters were hybridized to ${ }^{32} \mathrm{P}$-labelled probes derived from total cDNA from either the forward or reverse subtracted libraries using the PCR-Select differential screening kit (Clontech). The membranes were washed with low stringency $(2 \times$ SSC, $0.5 \%$ SDS; $3 \times, 20$ min each) and high stringency $(0.2 \times$ SSC, $0.5 \%$ SDS; $3 \times, 20$ min each) buffers at $65^{\circ} \mathrm{C}$. The radiolabelled DNA was detected using a PharosFX Molecular Imaging System (Bio-Rad).

\section{Identification of testis genes}

Plasmid DNA was recovered from selected bacterial colonies (strong signal with the forward and low signal with the reverse subtracted probe) and sequenced using Big 
Dye v3.1 chemistry (Applied Biosystems). DNA sequences were compared to the Ae. aegypti genome within the VectorBase database and predicted Drosophila melanogaster homologs were identified using BLAST-X against the nonredundant database at NCBI with default parameters.

Tissue and sex-specific expression of the identified genes was confirmed and quantified using qRT-PCR, using primers listed in Table 1, comparing expression of the genes' expression levels within testes, ovaries, and adult bodies minus the excised gonads. S7 ribosomal protein $(S 7 r p)$ gene expression was used as an internal reference to compare levels of RNAi. A single reference gene was deemed sufficient as the PCR efficiencies of the primer sets were calculated using the method of Pfaffl [22], and were found to be essentially equivalent for all genes targeted by RNAi (bol, fzo, gas 8 , nht, zpg) and for the $S 7 r p$ reference gene, with values ranging between 95.2-98.1\%. Melt curve analyses were also performed and confirmed that only a single product was amplified with each primer pair in every sample. Analysis of gene expression was performed using the $2^{-\Delta \Delta C t}$ method [23], comparing expression in specific dsRNA treated samples to gus-dsRNA treated samples.

\section{Isolation of the $d s x^{\mathrm{F}}$ gene fragment in Ae. aegypti}

The two primers pairs - dsxf1-for (GGTCAAGCCGTG GTCAATGAAT) and dsxf1-rev (CAACATTCTCCGCG CACAGG), and dsxf2-for (GCAAATGCTGTTTAAC GATAATAG) and dsxf2-rev (CGGAGCCGTTTGGC AACGG)- were used to amplify portions of the two female-specific exons of the $d s x^{\mathrm{F}}$ transcript (GenBank: DQ440532, GenBank:DQ440533). These two PCR products were subsequently used as templates to prepare dsRNAs by in vitro transcription, and were used in equimolar concentrations for dsRNA soaking or were cloned into pL4440 plasmids, to be used to transform E. coli, for bacterial feeding, as described below.

\section{Injecting dsRNA into mosquito pupae}

Total RNA was extracted from late pupae and early adult male $A e$ e aegypti, using QIAshredders (Qiagen) to homogenize tissues and an RNeasy RNA extraction kit (Qiagen). RNA was treated with amplification grade DNase I (Invitrogen) and $1 \mu \mathrm{g}$ was used to synthesize cDNA using a First Strand cDNA Synthesis kit (Invitrogen). The cDNA served as template DNA for PCR amplification of gene fragments ranging in size from 260 to $380 \mathrm{bp}$ in length, using the primers listed in Table 1. The gene fragments were subcloned into the cloning vector pDrive (Qiagen), and later excised from pDrive using either ApaI and PstI or MluI and NotI restriction enzymes, then ligated into a similarly-digested plasmid pL4440, a vector possessing convergent T7 promoters (kindly provided by Andrew Fire, Stanford University). A
$401 \mathrm{bp}$ fragment of the $\beta$-glucuronidase (gus) gene, a bacterial gene specific to E. coli, was amplified by PCR from the pBacPAK8-GUS plasmid (Clontech) using the following primers: GusF (CCCTTACGCTGAAGA GATGC) and GusR (GGCACAGCACATCAAAGAGA). The PCR product was cloned into the dsRNA transcription plasmid pL4440, as described above, to be used as a negative control. DNA templates for in vitro transcription of each of the gene fragments in pL4440 were PCR-amplified using the following pL4440specific primers: pL4440F (ACCTGGCTTATCGAA) and pL4440R (TAAAACGACGGCCAGT). PCR products were purified using a QIAquick PCR purification kit (Qiagen). The MEGAscript RNAi kit (Ambion) was then used for in vitro transcription and purification of dsRNAs. DsRNAs were diluted to $0.1 \mathrm{mg} / \mathrm{ml}$ in $20 \mathrm{mM}$ phosphate, $\mathrm{pH} 7$, and each pupa was injected with $50 \mathrm{nl}$. To assess for RNAi, insects were allowed to develop until 3-days post-eclosion and RNA was extracted and subjected to qRT-PCR analyses as described above.

\section{Introducing dsRNA to mosquito larvae}

a) DsRNA soaking method: Mosquito larvae (from $1^{\text {st }}$ to $4^{\text {th }}$ instars) were soaked in two concentrations of in vitro-transcribed dsRNA $(0.02$ and $0.2 \mathrm{mg} / \mathrm{ml}$ dsRNA) in dechlorinated tap water for $1 \mathrm{~h}$ /day, for 6 days, and were returned to their feeding trays after each dsRNA exposure. b) Feeding insect dsRNAexpressing bacteria: The pL4440 plasmids containing the mosquito genes were used to transform HT115 (DE3) E. coli cells and dsRNA production was induced by growing the liquid cultures of Luria Broth supplemented with $50 \mu \mathrm{g} / \mathrm{ml}$ ampicillin and $0.4 \mathrm{mM}$ IPTG. Once the cultures had reached an OD of $0.7-0.8$, the cells were pelleted by centrifugation and mixed with $1 \%$ LB-agar containing ampicillin and IPTG and $1 \mathrm{~g}$ of finely ground Tetramin fish flakes, cooled to $42^{\circ} \mathrm{C}$ to ensure the bacteria were not heat killed. The agar bacteria mixture was plated to a thickness of $5 \mathrm{~mm}$, cooled, and then cubed into $5 \mathrm{~mm}$ cubes, to be fed to mosquito larvae. To feed heat-killed bacteria in the same mixture, following pelleting, the bacteria were exposed to $70^{\circ} \mathrm{C}$ for $1 \mathrm{~h}$, and then mixed with the agar-Tetramin mixture and plated and cubed. To check that the bacteria were heat killed, $2 \mu \mathrm{l}$ of both heat-killed and non-heat killed bacteria were separately mixed with $50 \mu \mathrm{L} \mathrm{LB}$, and spread on LB plates, to check for bacterial growth. The agar cubes for mosquito feeding were stored at $4^{\circ} \mathrm{C}$ for up to 1 week before use. Mosquito larvae were raised in densities of 1 larva/2 ml water (groups of 10 larvae in $20 \mathrm{ml}$ ) and provided single cubes of bacteria-containing agar on a daily basis. As pupae developed, they were transferred to individual vials to await eclosion and sexsorting. 
Table 1 Primers used to amplify gene fragments for dsRNA synthesis or for qRT-PCR analysis

\begin{tabular}{|c|c|c|c|}
\hline Gene & Forward (F); Reverse (R) & dsRNA primers & qRT-PCR primers \\
\hline \multirow[t]{2}{*}{ AAEL001033 } & $\mathrm{F}$ & TTCAAGCAACCGGTGACAG & ACACTTCGCTCATTCCAC \\
\hline & $\mathrm{R}$ & TTGAGGGACGTTITGGAAGC & ACCTTGCTGTCTCCATCC \\
\hline \multirow[t]{2}{*}{ AAEL001156 } & $\mathrm{F}$ & GCAAAACTGACCATCCTGCA & CGATGTGGACTATACGGAAAC \\
\hline & $\mathrm{R}$ & CCGTTCTTGCAGTTTCAGCT & GGATTACTTGACGGTGCTTC \\
\hline \multirow[t]{2}{*}{ AAEL001684 } & $\mathrm{F}$ & CTGTGCCGGTTATTCAGCTC & GGTATTCGTCGGTGGTATCAG \\
\hline & $\mathrm{R}$ & GGGATGTTGTTGATCGTCGG & GCCTCGTGTTCGGTTTCG \\
\hline \multirow[t]{2}{*}{ AAEL002084 } & $\mathrm{F}$ & TTGCTGGACGAGAAGGAAGT & AAGGAGTACGAAGAGAAGAAG \\
\hline & $\mathrm{R}$ & ACTGAGCTGGTTGGTGAAGA & CGGAAGCGGTTCATTAGG \\
\hline \multirow[t]{2}{*}{ AAEL002275 } & $\mathrm{F}$ & AGATCGAGCACTGTTTCCGA & GTCGTTGGTGCGGCGTTG \\
\hline & $\mathrm{R}$ & CGTATACATGGGCCCGATCT & CCTTGTTGTCCTCCTCATCCTTGG \\
\hline \multirow[t]{2}{*}{ AAEL003501 } & $\mathrm{F}$ & CGCAAGGATCGGAAACCAAT & CAGCAGGATACGGTCTTC \\
\hline & $\mathrm{R}$ & CATGCTGTAGATCGGGTTGC & GAATAGGTCGGATTGTTGG \\
\hline \multirow[t]{2}{*}{ AAEL003757 } & $\mathrm{F}$ & AAAGGAGCGAAGGAGACCAA & GCGGCGGATGCGATTCTC \\
\hline & $\mathrm{R}$ & AGGTAGTGTTCAGGGCCTC & GACTCTTGGCGGAACGATAGC \\
\hline \multirow[t]{2}{*}{ AAEL004231 } & $\mathrm{F}$ & AGCCAAAGGAAGTACGGTCA & GAGCATCAGTCGCACATC \\
\hline & $\mathrm{R}$ & CTITCAGCTCTCCGATGTGC & TCCTTCTCTCGCAGTAACC \\
\hline \multirow[t]{2}{*}{ AAEL004471 } & $\mathrm{F}$ & CGCGCCAAGAAGAAGATCAA & AAGCACAACACCAGGAAC \\
\hline & $\mathrm{R}$ & ATITTGCCCGCAGCATAGC & GAAACCATCAGCCAAAGC \\
\hline \multirow[t]{2}{*}{ AAEL004696 } & $\mathrm{F}$ & GATACCGAAATGTGCACGCT & CCGTGCTTGAGTTGATAC \\
\hline & $\mathrm{R}$ & CCGGTTCTITGTCACTGCAA & ATTGGAATCTGATGGTGAG \\
\hline \multirow[t]{2}{*}{ AAEL004939 } & $\mathrm{F}$ & TATCCTGGGCAGCTGAACTC & GAACACCACCGCCATCAC \\
\hline & $\mathrm{R}$ & CAATGCGAGAAGGTACCGTG & CCTGCTTCTTCTTGACTTTCG \\
\hline \multirow[t]{2}{*}{ AAEL005010 } & $\mathrm{F}$ & GTITTCGTCGGTCCGGTTAG & CGGTGGAAGTGGATTGTC \\
\hline & $\mathrm{R}$ & TTGGCTTGGGTCTCCTTGAT & CGTTCTGATTCTTGCTGATG \\
\hline \multirow[t]{2}{*}{ AAEL005975 } & $\mathrm{F}$ & TGGACAAGGCGGAACAAAAG & GAGCAGAGATGGAGGAAC \\
\hline & $\mathrm{R}$ & CTTGATTCGAGGCCTCAACG & CAGGCGTAACAGTCGTAG \\
\hline \multirow[t]{2}{*}{ AAEL006726 } & $\mathrm{F}$ & GCATTCCTGTTCTCGTTCCC & TGGCTTTGGTTCATTGTG \\
\hline & $\mathrm{R}$ & TGAAGTCACATTTGGCCAGC & TATCCGATGTTGGCTTCC \\
\hline \multirow[t]{2}{*}{ AAEL006841 } & $\mathrm{F}$ & CATCGGGTGTTGCTTCTACG & ACGGTGCCTATCTGAGAAG \\
\hline & $\mathrm{R}$ & TCAAAGTACACGTGCTGCAG & GGATGCTGATGAACGCTAC \\
\hline \multirow[t]{2}{*}{ AAEL006975 } & $\mathrm{F}$ & GCCATTTCGATGCCAAAACG & CGTCTGGTGTAGGTGCTAAGTG \\
\hline & $\mathrm{R}$ & TCGACTGAAATCCGGGAACA & TGCTTGTTCTGCCGCTTGC \\
\hline \multirow[t]{2}{*}{ AAEL007144 } & $\mathrm{F}$ & AACACTTCAACACGTGTCGG & TTCGCATACGGAGTGTTAC \\
\hline & $\mathrm{R}$ & TCGTACACTTCAGCGGAGTT & CCTTGTGGATGTAGTCTCG \\
\hline \multirow[t]{2}{*}{ AAEL007188 } & $\mathrm{F}$ & GCAGCGCCAATATCTGAACA & TTCGCAGTCGGATTACTTCTTC \\
\hline & $\mathrm{R}$ & TTCCCGCTTCTTCAGGTGAT & TGGTTCTTGGTGATATTCGTAGC \\
\hline \multirow[t]{2}{*}{ AAEL007434 } & $\mathrm{F}$ & CTGTCCTCGCCCAATGAATG & TGCGTTCTGTTCATAATGGTTAC \\
\hline & $\mathrm{R}$ & CTGCAGTAAATCTCCGCACC & GTCGGGTTTGGTTTCACTCC \\
\hline \multirow[t]{2}{*}{ AAEL007544 } & $\mathrm{F}$ & ATCGTCTATGGCCGGCTTTA & AGTTCTCCTTCCGACATC \\
\hline & $\mathrm{R}$ & TAGCGCTATGATGTCGTCGT & GTAAGCCGCACATTCATC \\
\hline \multirow[t]{2}{*}{ AAEL007684 } & $\mathrm{F}$ & AGCGATGCAGGACGAGATTA & CGCCACCAGATGTCCTAATG \\
\hline & $\mathrm{R}$ & CGTGGGCCAGTTTCTTATCG & CAGTTGTTCCGATTGCTTCC \\
\hline \multirow[t]{2}{*}{ AAEL008428 } & $\mathrm{F}$ & TTGGGCATGCTTCACTGATG & TGTTGGATGATGTTGTGAGATGTG \\
\hline & $\mathrm{R}$ & ATCGTCGGAGTATCGCTGTT & ATCGTCGGAGTATCGCTGTTC \\
\hline
\end{tabular}


Table 1 Primers used to amplify gene fragments for dsRNA synthesis or for qRT-PCR analysis (Continued)

\begin{tabular}{|c|c|c|c|}
\hline \multirow[t]{2}{*}{ AAEL008678 } & $\mathrm{F}$ & GCCGTTTCCAGGACAACTTT & CCAGTCAGAGGGCGAATG \\
\hline & $\mathrm{R}$ & GTAGTAATCCCGCTCTGCCT & CTTCTCCGTCAGGTCATCC \\
\hline \multirow[t]{2}{*}{ AAEL009047 } & $\mathrm{F}$ & GGAACGGTGAAATCGATGGG & CACAGAGGAGGAAGTAATTG \\
\hline & $\mathrm{R}$ & TTCACTGCTGTCGTTGTGTG & CACTATTGGACTGCTAACG \\
\hline \multirow[t]{2}{*}{ AAEL009321 } & $\mathrm{F}$ & CAGCGACGAACCACAATGTA & GTATATGTCGCCTCGGTTC \\
\hline & R & GCTTATCGCCGATGGTTACC & GGGTTGTATCGGTGTTCC \\
\hline \multirow[t]{2}{*}{ AAEL009357 } & $\mathrm{F}$ & TCAAGCAAGTGCTGGACAAC & Not completed \\
\hline & $\mathrm{R}$ & TGCCTTCAGGTCGTTCTCTT & \\
\hline \multirow[t]{2}{*}{ AAEL009553 } & $\mathrm{F}$ & CAGTAGCTITCCGTCCATGC & GTTGCTCTTCGTCATTATTCC \\
\hline & $\mathrm{R}$ & GACCAGCGGATAAATCGCAG & AAATCCATTGCCATCTTTGC \\
\hline \multirow[t]{2}{*}{ AAEL010639 } & $\mathrm{F}$ & GCGCACGTTATGATGGGAAT & GCGGAGATGTTACCTTGAAG \\
\hline & $\mathrm{R}$ & TTCCCTTGCATCACATCCCT & GAAACTACCTGGACCTCTGG \\
\hline \multirow[t]{2}{*}{ AAEL011098 } & $\mathrm{F}$ & TTGGCGAAATTCTGCAAGCT & ACTCATACACTCGTTTCAAG \\
\hline & R & AGCCGAAACGTTTGCTTCAT & TCCATATCCGAAGCACTC \\
\hline \multirow[t]{2}{*}{ AAEL011310 } & $\mathrm{F}$ & GGATATGAGACCCGAACCGT & GAAGATGCCGAGCGAGAG \\
\hline & $\mathrm{R}$ & GTITCTTCGGTGCCTTCGT & GACCGACCTGGATGGATTC \\
\hline \multirow[t]{2}{*}{ AAEL012096 } & $\mathrm{F}$ & AATACCAGCACGCTCTCTCA & Not completed \\
\hline & $\mathrm{R}$ & ATTGCATCGGTGGCAAGTTT & \\
\hline \multirow[t]{2}{*}{ AAEL012446 } & $\mathrm{F}$ & GCTACTTGGATTTGGGCGAC & CGGAGCCAAGGAGGTCATC \\
\hline & $\mathrm{R}$ & ATCGCTTCGGACAGGATGAT & ACAGCAGCAGAAGCAGAGG \\
\hline \multirow[t]{2}{*}{ AAEL013621 } & $\mathrm{F}$ & TाTGAACCCGGAAAAGGCAG & CCAGAGCAACCGAGAGTATG \\
\hline & $\mathrm{R}$ & TTCGACGAAATCCTCCCACA & CGACGAAATCCTCCCACAG \\
\hline \multirow[t]{2}{*}{ AAEL013723 } & $\mathrm{F}$ & GATGAAACTGCCGCCACTAG & TTAAGATTGTCACCTTCACC \\
\hline & $\mathrm{R}$ & TTGCCGAACCGTTGGAAAAT & CGTTGTAGATGTTCTGTCC \\
\hline \multirow[t]{2}{*}{ AAEL013737 } & $\mathrm{F}$ & AAGACTTGGGAAGAGGACGG & TGTAGTTCGGTATCGTTCGG \\
\hline & $\mathrm{R}$ & TTCTCTAGCAGCTGGATCCG & TCGGCATTCCTTCGTTCG \\
\hline \multirow[t]{2}{*}{ AAEL014067 } & $\mathrm{F}$ & TTGCTCATACGCTCCATTGC & ACAACAGAGCCTAAGACTATC \\
\hline & $\mathrm{R}$ & TTGCTCCTGAACGGTGAGAT & CGACAATCATATTCTCACAGC \\
\hline \multirow[t]{2}{*}{ AAEL014408 } & $\mathrm{F}$ & GACCGATCCTGCAAAAGTCC & TGGACGATAATGCTCAAC \\
\hline & $\mathrm{R}$ & TTGGTCCTGGGTGTAGAGG & GAGGCGAATGGAGTTATG \\
\hline
\end{tabular}

\section{Mosquito fertility assays}

Individual 3-day old dsRNA-treated mosquitoes were provided virgin mates in $25 \mathrm{ml}$ plastic vials for 2 days. Mosquitoes were provided $10 \%$ sucrose ad libitum, and to assess fertility and fecundity, females were provided blood meals (derived from rats) 2 days post-mating, and eggs were collected on moistened paper towels placed within the vials for between a week and 10 days postblood meal. Eggs were incubated at $25^{\circ} \mathrm{C}$ for 4 days, and then immersed in water to assess hatch rates and calculate fecundity. If no eggs were produced, or all eggs failed to hatch, the dsRNA-treated insect was considered sterile.

For small population mating competitions involving only 20 mosquitoes, $500 \mathrm{ml}$ glass jars with screencovered lids were used, while mating competitions using
200 mosquitoes were conducted in larger (45 X $45 \mathrm{x}$ $45 \mathrm{~cm}$ ) screened cages. All sterility and fecundity values for dsRNA-treated insects were expressed as percentages, relative to negative control animals, which were considered as $0 \%$ sterile, $100 \%$ fecund.

\section{Statistical analysis}

For every experiment, sample size was determined empirically (preliminary experiments were performed) to ensure that the desired statistical power could be achieved. The values are expressed as mean \pm s.e.m. The error bars (s.e.m.) shown for the results were derived from biological replicates, not technical replicates. Sterility and fecundity values that were expressed as percentages, relative to the values obtained from negative control treatments, were arcsine 
transformed to produce values that displayed a normal distribution and comparable variance. Significant differences between two groups were then evaluated using a two-tailed, unpaired $t$-test.

\section{Results \\ Identification of testes-specific genes}

To test whether RNAi could be used to develop an effective SIT technology, we used a suppression subtractive hybridization technique [24] to identify genes that were preferentially or exclusively expressed in Ae. aegypti testes. A testis-specific cDNA library was prepared by first hybridizing testis cDNA with cDNA derived from the rest of the body. Amplification of hybrids corresponding to common sequences was suppressed, yielding a library enriched for differentially expressed sequences within the testes. A total of 37 genes were identified that were predominantly or exclusively expressed in male Ae. aegypti testes. Reverse transcriptase-PCR (RT-PCR) confirmed that all 37 genes were expressed in the testes, with 15 genes showing no evidence of expression in larval stages, other adult tissues, nor in female ovaries. Six genes were expressed in both testes and ovaries, but not in other adult tissues. Homologs for all but a few of the genes were identified in Drosophila melanogaster, and many were similarly expressed in either testes or ovaries (Table 2), providing more than a dozen target genes to use.

\section{Development of RNAi-induced sterile and low fertility males}

A subset of 10 genes was selected for RNAi-mediated knockdown to determine if they would render males sterile. In addition to sterilizing the males, the knockdown of the target gene should not adversely affect the male's ability and willingness to mate, as males must still compete effectively with wild, fertile males. For this reason, we selected genes that were not expressed in other tissues of the male, thus avoiding any adverse effects on mating behaviors or physiologies. DsRNAs targeting the 10 genes were injected into pupae, and subsequent quantitative RT-PCR (qRT-PCR) confirmed that each of the 10 transcripts were knocked down by between 70 95\% within the adult males four days' post-injection. The dsRNA-injected males were then mated with virgin females, and most (9/10) of the dsRNAs induced complete sterility in more than half of the injected males, and substantially reduced fecundity in the others, relative to either non-injected males or to males injected with an E. coli-specific dsRNA (Figure 1A).

Mating competiveness of the sterilized males was also assessed by combining them with untreated males and virgin females in small population cages. When the number of progeny was scored, five dsRNAs both induced male sterility and caused significant reductions $(>75 \%)$ in the number of viable progeny produced (Figure 1B). These particular dsRNAs all targeted mosquito genes that had homologs in D. melanogaster and were known to be involved in spermatogenesis: boule (bol); fuzzy onions (fzo); growth arrest specific protein 8 (gas8); no-hitter (nht); and zero population growth $(z p g)$. One of the genes, $z p g$, had previously been identified as important for male fertility in the malaria vector An. gambiae: dsRNA targeting zpg transcripts injected into An. gambiae embryos produced males that lacked sperm, but they could still transfer male accessory gland fluids to the females to induce female post-mating behaviors, including oviposition of unfertilized eggs [25].

The other five dsRNAs tested in this screen produced sterile or partially sterile males that were not strongly competitive, allowing the females to continue to mate with fertile males. While these dsRNAs had targeted genes that were apparently expressed exclusively in the testis, their knockdown may nevertheless have affected male mating performance by reducing the male's mating activity, sperm transfer, or transfer of male accessory gland proteins - some of which can affect female mating behavior and fecundity [26].

The five spermatogenesis-specific dsRNAs were subsequently administered to mosquito larvae by soaking larvae in dsRNA solutions (at two concentrations: $0.02 \mathrm{mg} /$ $\mathrm{ml}$ and $0.2 \mathrm{mg} / \mathrm{ml}$ ) for one hour each day for six days. At the higher concentration, these brief exposures to the dsRNAs sufficiently induced sterility in most (72-92\%) of the males (Figure 2A). The small number of fertile males had severely reduced fecundity. The lower concentration of dsRNAs produced fewer sterile males (20-35\%) but, encouragingly, by mixing low concentrations of different dsRNAs together, sterility frequencies could be enhanced to nearly $100 \%$ (Figure 2A). This suggests that combinations of dsRNAs may permit lower concentrations of dsRNAs to be used for sterile male production. In these dsRNA soaking treatments, the dsRNA likely entered the insects by ingestion, although entry through other routes (e.g. cuticular penetration) cannot be excluded. Nevertheless, RNAi clearly spread beyond the initial entry points to reach the testes, and this relatively simple "bathing", or soaking, technique underscores one of the many advantages of this new transformative approach to SIT.

\section{Bacterial dsRNA mosquito larval treatment}

Mosquito larvae were fed E. coli bacteria expressing dsRNAs, in a manner similar to that used to feed Caenorhabditis elegans nematodes [27]. Both live and heat-killed E. coli were embedded into agar cubes containing finely ground larval diet, on which the mosquito larvae grazed until pupation. Three of the five E. coli strains expressing dsRNA sterilized more than $60 \%$ of 
Table 2 Genes expressed predominantly or strictly in the testis of Ae. aegypti

\begin{tabular}{|c|c|c|c|c|}
\hline Aedes aegypti accession \# & D. melanogaster homolog & Testis-specific? & Expressed in larvae? & Ovary-specific? \\
\hline AAEL001033 & CG8208 (MDB-like) & No & Yes & Yes \\
\hline AAEL001156 & CG5280 & Yes & No & No \\
\hline AAEL001684 & CG4727 (bol) & Yes & No & No \\
\hline AAEL002084 & CG14220 & Yes & No & Yes \\
\hline AAEL002275 & CG3565 & Yes & No & No \\
\hline AAEL003501 & CG10252 & Yes & Yes & No \\
\hline AAEL003757 & CG4434 & No & Yes & No \\
\hline AAEL004231 & CG14271 (Gas8) & Yes & No & No \\
\hline AAEL004471 & CG4568 (fzo) & Yes & No & No \\
\hline AAEL004696 & CG5737 & No & Yes & No \\
\hline AAEL004939 & CG32396 (ß-tub) & Yes & No & No \\
\hline AAEL005010 & CG14305 & Yes & No & No \\
\hline AAEL005975 & CG15259 (nht) & Yes & No & No \\
\hline AAEL006726 & CG6647 (zpg) & Yes & No & Yes \\
\hline AAEL006841 & - & Yes & No & Yes \\
\hline AAEL006975 & CG18369 (S-Lap5) & Yes & No & No \\
\hline AAEL007144 & CG12423 (k/h/10) & No & No & No \\
\hline AAEL007188 & CG17083 & Yes & No & No \\
\hline AAEL007434 & - & Yes & No & No \\
\hline AAEL007544 & CG10895 (lok) & Yes & No & Yes \\
\hline AAEL007684 & CG4767 (tek) & No & Yes & No \\
\hline AAEL008428 & CG10841 & No & No & No \\
\hline AAEL008678 & CG18190 & No & Yes & Yes \\
\hline AAEL009047 & CG8819 (achi) & No & Yes & Yes \\
\hline AAEL009321 & - & No & Yes & Yes \\
\hline AAEL009357 & CG2146 & No & Yes & Yes \\
\hline AAEL009553 & CG12813 & No & Yes & No \\
\hline AAEL010639 & CG5458 & Yes & No & No \\
\hline AAEL011098 & CG8362 & Yes & No & Yes \\
\hline AAEL011310 & CG9313 & Yes & No & No \\
\hline AAEL012096 & CG18472 & Yes & No & No \\
\hline AAEL012446 & CG6303 & No & Yes & Yes \\
\hline AAEL013621 & CG17564 & Yes & Yes & No \\
\hline AAEL013723 & CG31000 (heph) & Yes & Yes & Yes \\
\hline AAEL013737 & CG6971 & Yes & No & No \\
\hline AAEL014067 & CG5048 & Yes & Yes & No \\
\hline AAEL014408 & CG4965 (twe) & Yes & No & Yes \\
\hline
\end{tabular}

Genes were identified in a SSH screen, selectively amplifying genes expressed in testes relative to the rest of the male's body. cDNA was extracted from dissected male testes, the male body minus testes, $4^{\text {th }}$ instar larvae, dissected female ovaries, and female bodies minus ovaries, and RT-PCR was then used to assess whether the genes were 1) testis-specific (i.e. in testes, but not other adult tissues); 2) expressed in larvae; and 3) ovary specific (i.e. in ovaries, but not other female tissues). Genes are ordered in ascending VectorBase (www.vectorbase.org) accession numbers.

the males, and by combining different dsRNAexpressing E. coli strains, up to $92 \%$ sterility could be achieved (Figure 2B). No significant loss of potency of the RNAi effect occurred when the bacteria were heatkilled (Figure 2B).

\section{Biasing emerging adults towards males}

To produce a male-biased population of mosquitoes, and obviate the need for later sex-sorting, we also attempted to silence a female differentiation gene. In many insects, the doublesex $(d s x)$ gene is alternatively 


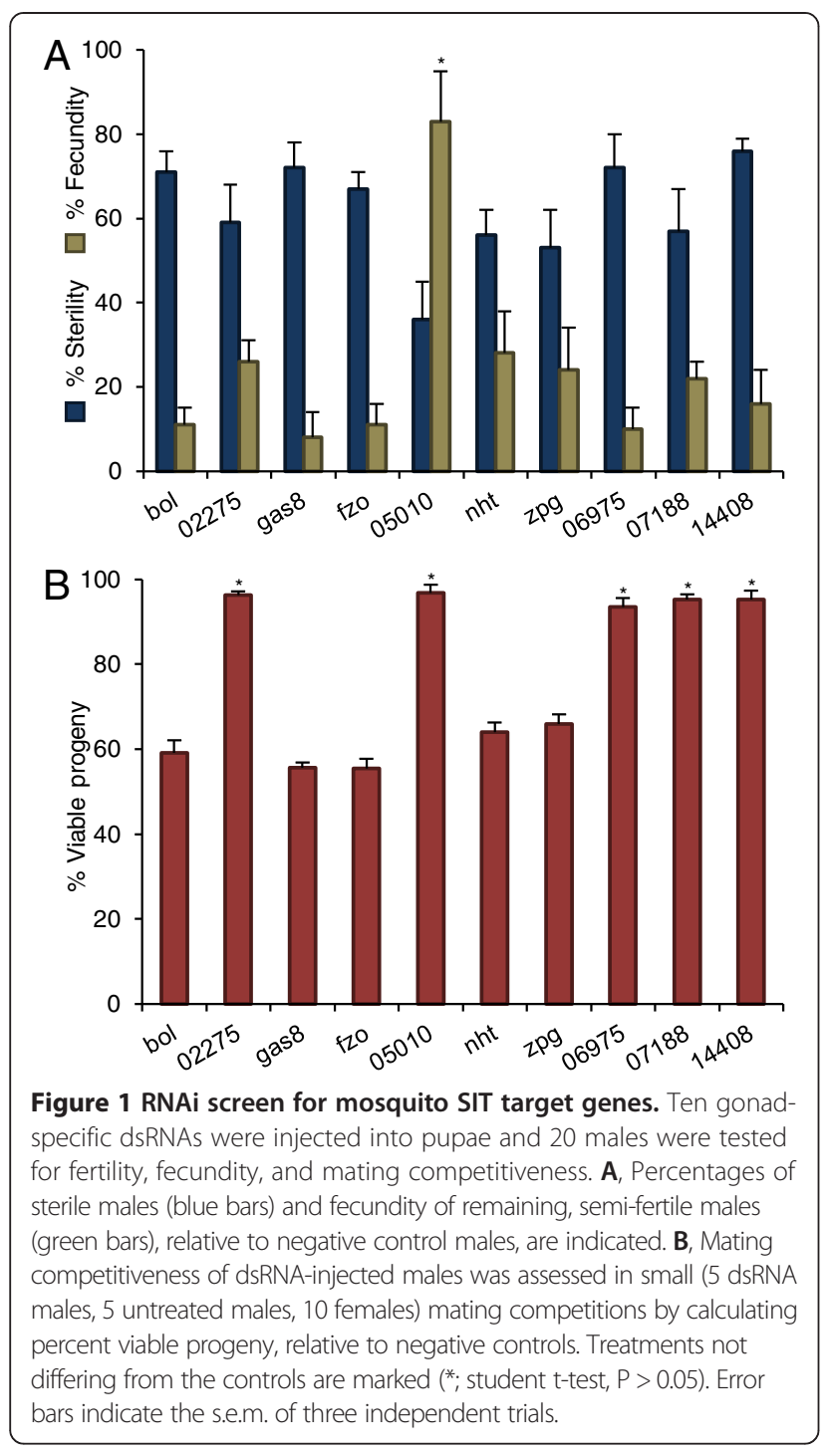

spliced in males and females, with the female splice variant of $d s x$ producing $\mathrm{DSX}^{\mathrm{F}}$, which acts as a transcription factor that regulates many female-specific genes throughout an insect's development and in tissues that display sexual dimorphic development [28]. RNAi-mediated knockdown of the female splice variant of $d s x\left(d s x^{\mathrm{F}}\right)$ was achieved by either soaking the mosquito larvae in dsRNA, or by feeding the larvae E. coli expressing dsRNA that targeted the two female-specific exons of Ae. aegypti $d s x$ [29]. Both dsRNA delivery methods resulted in a strongly male-biased population of insects (up to 97\% males; Table 3). The fact that the number of dsRNA-treated larvae that developed into adults was virtually halved relative to the negative controls, with no significant increase in males (Chi-square test, $\mathrm{P}>0.05$ ), suggests that the vast majority of RNAi-affected females simply failed to develop, rather than having undergone a sex-reversal due to the lack of $d s x^{\mathrm{F}}$. Although a complete lack of females remains preferable for any SIT approach, the few extant females produced were either sterile, or failed to bloodfeed, mate or survive, and hence, should not compromise the efficacy of this technique.

\section{Mating competitions with RNAi-induced sterile and low fertility males}

When dsRNA targeting male-specific gas 8 was combined with dsRNA targeting $d s x^{\mathrm{F}}$ and fed to Ae. aegypti larvae, the mosquitoes that developed were almost entirely males (96\%), and the vast majority of these (96\%) were sterile. The remaining $4 \%$ of males were only partially fertile, producing, on average, less than $20 \%$ of the progeny produced by fertile males. We then mixed the dual dsRNA-treated mosquitoes in varying proportions with untreated male mosquitoes in population cages holding 100 untreated females. These mating competition experiments suggested that the dsRNA-treated males were very effective competitors, as significant reductions in progeny were observed when the populations were seeded with $25 \%$ dsRNA-treated males (ANOVA, $\mathrm{P}<0.01$ ) (Figure 3); at 50\% dsRNA-treated males, $50 \%$ reduction in progeny was observed. At higher sterile male seeding densities, very strong population suppression was observed, ranging from 95 to $100 \%$. Even without completely eliminating the few contaminating females from the dsRNA-treated insects, the sterilized males were clearly good competitors and proved effective at reducing mosquito populations in these cage settings.

\section{Discussion}

This study demonstrates that it is possible to generate non-transgenic, non-radiation sterile or low fertility male mosquitoes that efficiently mate with wild females, thus preventing the production of offspring and reducing the population size. Using the dengue vector Ae. aegypti as a model system, dsRNAs were introduced to mosquito larvae to induce an RNAi-mediated knockdown of male fertility genes and a female sex determination gene, overcoming the need to sex-sort insects to produce a malebiased, substantially sterile adult population.

These outcomes are both essential and exciting in terms of designing efficient SIT approaches in the future. They build on previous mosquito population suppression techniques, which release sterile male insects to seek out and mate with females, a proven biological control method for insects $[14,30]$. The RNAi-based approach, however, offers some unique adaptations to conventional SIT, including the potential for species-specificity of the dsRNA-based sterilizing molecules, avoiding the debilitating effects of radiation, and eliminating the need to sex-sort the insects before release. The RNAi effect following feeding on dsRNAs was remarkably robust and persistent throughout 

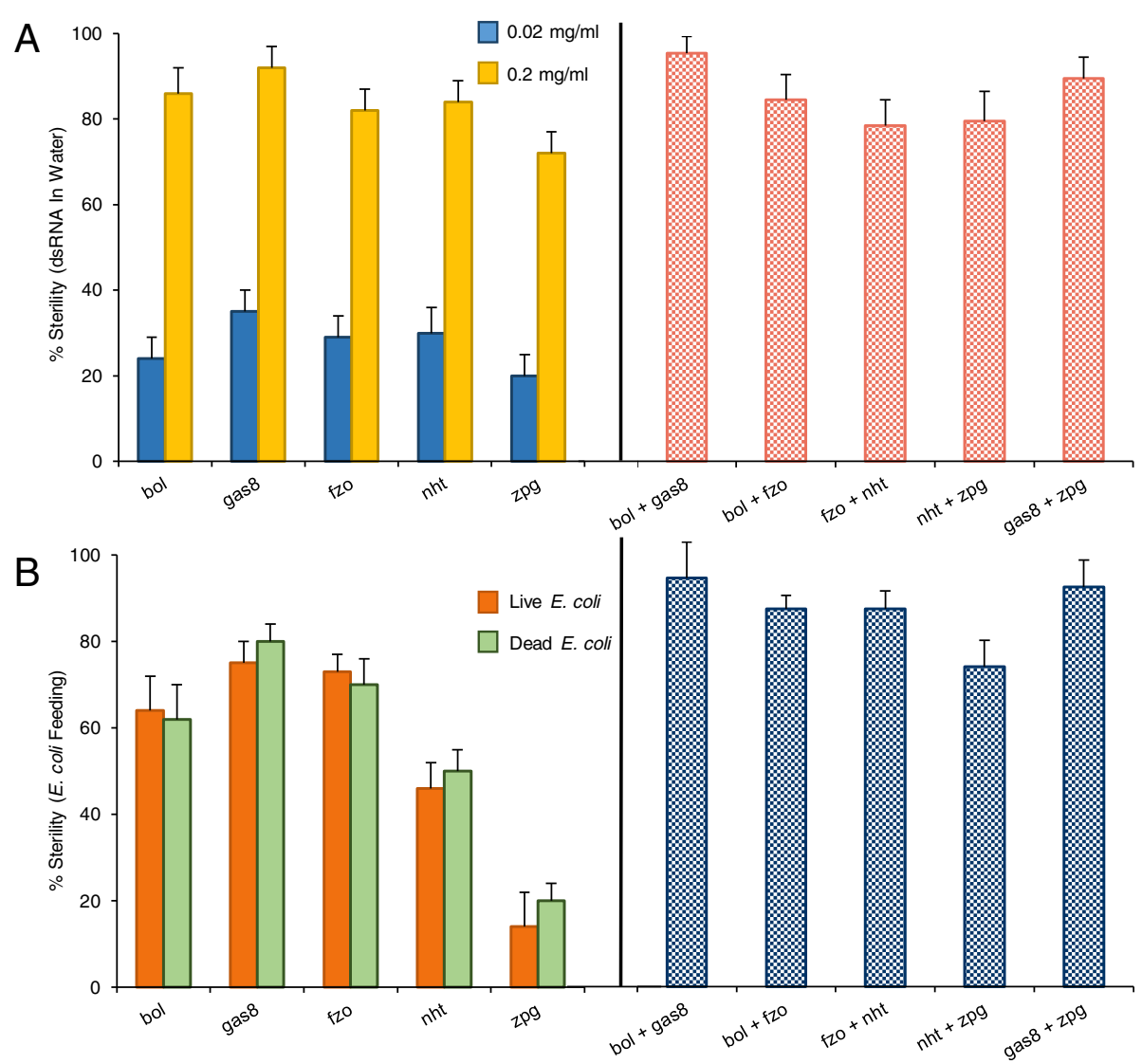

Figure 2 Feeding dsRNAs to larvae induces adult male sterility or low fertility. A, 100 larvae (from 1st to 4th instars) were soaked for $1 \mathrm{~h}$ each day for 6 consecutive days in two concentrations of five dsRNAs (left panel) or in mixtures of two dsRNAs (each $0.01 \mathrm{mg} / \mathrm{ml}$, right panel); the percentage of sterile males, relative to untreated controls, are indicated. B, 100 larvae were fed either live or heat-killed bacteria expressing one dsRNA (left panel) or mixtures of live E. coli expressing two dsRNAs (right panel), and the percent of sterile males, relative to untreated males, was assessed. Error bars indicate the s.e.m. of five independent trials. All dsRNA treatments induced significant reductions in fertility (ANOVA, $P<0.01$ ).

the development of the mosquitoes - a phenomenon that has been observed in a variety of insect species [31-33]. Exposure of the larvae to the testis-specific dsRNAs before and during early testis development was sufficient to cause adult sterility or very low fertility. Similarly, exposure to the $d s x^{\mathrm{F}}$ dsRNA was sufficient to inhibit complete development of females. While $d s x$ is absolutely required for the early stages of sex differentiation during embryonic development, it is also required for proper differentiation and development of the adults [28]. Homologs for $d s x$ have already been identified in Aedes and Anopheles mosquitoes $[34,35]$, and we anticipate that many of the genes identified as effective dsRNA-sterilizing targets by this study will have homologs in other insects. In our estimation, there is considerable potential to apply this novel technology to other insects amenable to SIT approaches, including important agricultural and human pathogen vectors.

Table 3 Treatment of mosquito larvae with $d s x^{\mathrm{F}}$-dsRNA reduced production of females and all were sterile

\begin{tabular}{llllll}
\hline dsRNA & dsRNA delivery & \# Larvae treated $^{3}$ & Females/males that developed & \# Females that blood fed & \# Females that produced progeny $^{4}$ \\
\hline gus & Daily soakings $^{1}$ & 420 & $207 / 200$ & 162 & 139 \\
dsx $^{F}$ & Daily soakings $^{1}$ & 440 & $6 / 242$ & 0 & 0 \\
gus & Larval feeding $^{2}$ & 445 & $238 / 187$ & 194 & 172 \\
dsx $^{F}$ & Larval feeding $^{2}$ & 460 & $7 / 241$ & 1 & 0
\end{tabular}

${ }^{1}$ Larvae $\left(1^{\text {st }}-4^{\text {th }}\right.$ instars) were soaked for $1 \mathrm{~h} /$ day for 6 days in $0.2 \mathrm{mg} / \mathrm{ml} \mathrm{dsRNA}$.

${ }^{2}$ Larvae were fed heat-killed E.coli continuously.

${ }^{3}$ Mixed sexes of larvae were treated.

${ }^{4}$ Females were provided 2 males for a period of one week, offered blood meals, and hatching of eggs monitored. 


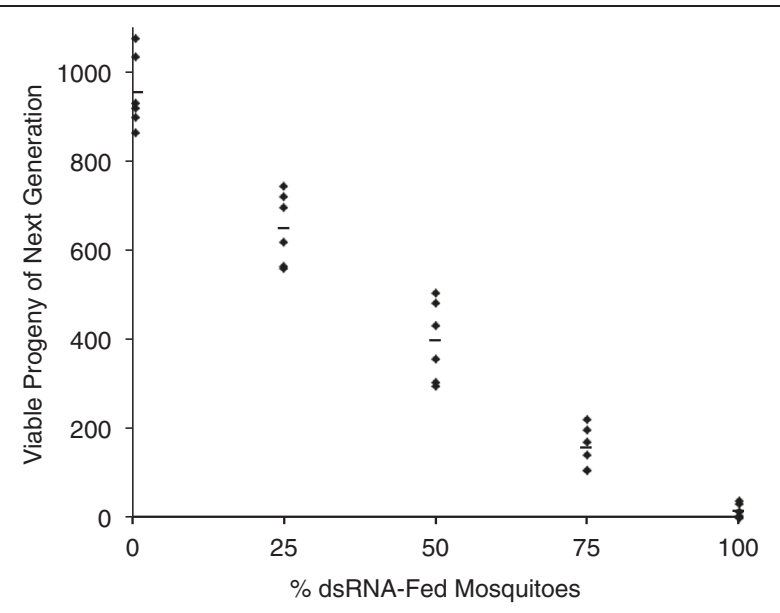

Figure 3 Seeding populations with dsRNA-treated mosquitoes reduces production of progeny. Larvae (first to fourth instars) were soaked daily for $1 \mathrm{~h}$ for 6 consecutive days in a mixture of $d s x^{F}$ and gas 8 -dsRNAs $(0.2 \mathrm{mg} / \mathrm{ml}$ each). The adults arising from these treatments were mixed at different proportions with untreated males (100 males in total), and were mixed with 100 virgin females. The number of viable progeny from each mating cage was assessed 10 days later. Each point represents a single cage experiment (i.e. 6 replicates for each treatment), with the average number of progeny indicated (horizontal lines). Progressive increases in sterile male seeding density produced significantly greater reductions in the number of progeny produced (ANOVA, $P<0.01$ ).

This RNAi method could be applied to both massreared insects and on insects collected from the field, which could be used to supplement laboratory-bred populations to counter possible declines in male vigor due to chronic inbreeding [36,37], or to minimize assortative mating behaviors that females could exhibit when exposed to mass-reared males [38]. While it is anticipated that RNAi technology could be easily adapted for insect mass-rearing facilities, the method also has the potential for field-based control via administration of either dsRNA-baited food sources or artificial larval environments. These approaches would see only competitive sterile or low fertility males emerge from eggs oviposited into the larval site and could potentially drive a local population to extinction.

In terms of the economics of this approach, our method of producing dsRNAs by in vitro transcription would be prohibitively expensive to treat large numbers of mosquitoes ( $\$ 200$ to produce $1 \mathrm{mg}$ of dsRNA to treat 100 larvae for a single experimental trial), but recent advances in large-scale ( $\mathrm{kg}$ quantities) production of dsRNAs [39] will greatly reduce costs. Production of dsRNA in bacteria [40] or other microorganisms [41] is a very cheap method of producing dsRNA and interestingly, heat-killed bacteria were just as effective as live bacteria at inducing RNAi in the mosquitoes. From a regulatory perspective, heat-killed bacteria may prove safer in terms of possible distribution of dsRNA into natural or artificially-constructed mosquito larval sites. For some species, the use of RNA microcarriers, such as liposomes [20] or nanoparticles [21] might be required to facilitate uptake of the dsRNA and/or stabilize the dsRNA in the larval environment.

While many of the genes identified in this study are shared with other insects, RNAi has an in-built sequence-specificity $[42,43]$, which, with careful design of the dsRNAs through knowledge of the gene sequences of sympatric species, could be developed to specifically target pathogen-transmission or pest species without impacting non-target species. This ability enhances the appeal of this new insect control technique, addressing yet another point of concern in earlier approaches in terms of impacts on other species in general and on beneficial species in particular.

\section{Conclusions}

Releasing sterile male insects to mate with wild females is an established method of insect population control. Previous technologies have been hampered by their need for radiation (that reduces male fitness) or genetic modification (with its risks and regulatory issues) to generate the sterile males. Here, we demonstrate production of non-radiated, non-transgenic sterile or extremely low fertility males of the dengue vector Ae. aegypti. By feeding dsRNA to the insects at larval stages, sperm production in adult males can be halted (producing fit substantially-sterile males) with a bias towards males emerging as adults, which avoids sex sorting. Our species-specific sequenced-based targets are common in other insects and may thus open a new industry in vector and pest control.

\section{Abbreviations \\ dsRNA: Double-stranded RNA; RNAi: RNA interference; GM: Genetically- modified; SIT: Sterile insect technique; dsx: Doublesex; bol: Boule; fzo: Fuzzy onions; gas8: Growth arrest specific protein 8; nht: No-hitter; zpg: Zero population growth; qRT-PCR: Quantitative reverse transcriptase-polymerase chain reaction.}

\section{Competing interests}

The authors declare that they have no competing interests.

\section{Authors' contributions}

SW designed the experiments; CNGE, ALP, ADS, and RC performed experiments and analysed data; SW and NWB wrote the paper. All authors read and approved the final version of the manuscript.

\section{Acknowledgements}

This study was supported by a grant from the Natural Sciences and Engineering Research Council to SW, and CNGE and ALP were supported by NSERC graduate scholarships.

\section{Author details}

${ }^{1}$ Department of Biological Sciences, University of Manitoba, Winnipeg, MB, Canada. ${ }^{2}$ School of Biological Sciences, University of Queensland, Brisbane, Australia. ${ }^{3}$ CSIRO Biosecurity Flagship, Brisbane, Queensland, Australia. 
Received: 7 January 2015 Accepted: 5 February 2015

Published online: 12 February 2015

\section{References}

1. McGraw EA, O'Neill SL. Beyond insecticides: new thinking on an ancient problem. Nat Rev Microbiol. 2013;11:181-93.

2. Knipling EF. Sterile-male method of population control. Science. 1959;130:902-4.

3. Knipling EF. The eradication of the screwworm fly. Sci Am. 1960;203:4-48.

4. Vreysen MJ, Saleh KM, Ali MY, Abdulla AM, Zhu ZR, Juma KG, et al. Glossina austeni (Diptera: Glossinidae) eradicated on the island of Unguja, Zanzibar, using the sterile insect technique. J Econ Entomol. 2000;93:123-35.

5. Shelly TE, Whittier TS, Kaneshiro KY. Sterile insect release and the natural mating system of the Mediterranean fruit fly, Ceratitis capitata (diptera: tephritidae). Ann Entomol Soc Am. 1994;15:470-81.

6. Bloem KA, Bloem S. SIT for codling moth eradication in British Columbia, Canada. In: Tan K-H, editor. Area-Wide Control of Fruit Flies and Other Insect Pests. Joint Proceedings of the International Conference on Area-wide Contro of Insect Pests, and the Fifth International Symposium on Fruit Flies of Economic Importance, May-June 1998. Pulau Penang: Penerbit Universiti Sains Malaysia; 2000. p. 207-14.

7. Helinski ME, Knols BG. Mating competitiveness of male Anopheles arabiensis mosquitoes irradiated with a partially or fully sterilizing dose in small and large laboratory cages. J Med Entomol. 2008;45:698-705.

8. Proverbs MD. Induced sterilization and control of insects. Annu Rev Entomol. 1969:14:81-102

9. Franz G, Gencheva E, Kerremans P. Improved stability of genetic sexseparation strains for the Mediterranean fruit fly. Ceratitis capitata Genome 1994:37:72-82.

10. Benedict MQ, Robinson AS. The first releases of transgenic mosquitoes: an argument for the sterile insect technique. Trends Parasitol. 2003;19:349-55.

11. Windbichler N, Papathanos PA, Crisanti A. Targeting the X chromosome during spermatogenesis induces $Y$ chromosome transmission ratio distortion and early dominant embryo lethality in Anopheles gambiae. PLoS Genet. 2008;4:e1000291.

12. Galizi R, Doyle LA, Menichelli M, Bernardini F, Deredec A, Burt A, et al. A synthetic sex ratio distortion system for the control of the human malaria mosquito. Nature Comm. 2014;5:3977.

13. Labbe GMC, Scaife S, Morgan SA, Curtis ZH, Alphey L. Female-specific flightless (fsRIDL) phenotype for control of Aedes albopictus. PLoS Negl Trop Dis. 2012;6:e1724.

14. Alphey L, Benedict M, Bellini R, Clark GG, Dame DA, Service MW, et al. Sterile-insect methods for control of mosquito-borne diseases: an analysis. Vector Borne Zoonotic Dis. 2010;10:295-311.

15. Phuc HK, Andreasen MH, Burton RS, Vass C, Epton MJ, Pape G, et al. Lateacting dominant lethal genetic systems and mosquito control. BMC Biol. 2007:5:11.

16. Harris AF, McKemey AR, Nimmo D, Curtis Z, Black I, Morgan SA, et al. Successful suppression of a field mosquito population by sustained release of engineered male mosquitoes. Nature Biotech. 2012;30:828-30.

17. Black WC, Alphey L, James AA. Why RIDL is not SIT. Trends Parasitol. 2011;27:362-70

18. Brown DM, Alphey LS, McKemey A, Beech C, James AA. Criteria for identifying and evaluating candidate sites for open-field trials of genetically engineered mosquitoes. Vector Borne Zoonotic Dis. 2014;14:291-9.

19. Hannon GJ. RNA interference. Nature. 2002;418:244-51.

20. Singh AD, Wong S, Ryan CP, Whyard S. Oral delivery of double-stranded RNA in larvae of the yellow fever mosquito. Aedes aegypti: implications for pest mosquito control J Insect Sci. 2013;13:69.

21. Zhang X, Zhang J, Zhu KY. Chitosan/double-stranded RNA nanoparticlemediated RNA interference to silence chitin synthase genes through larval feeding in the African malaria mosquito (Anopheles gambiae). Insect Mol Biol. 2010;19:683-93.

22. Pfaffl MW. A new mathematical model for relative quantification in real-time RT-PCR. Nucl Acids Res. 2001;29:e45

23. Livak KJ, Schmittgen TD. Analysis of relative gene expression data using real-time quantitative PCR and the 2(-Delta Delta C(T)) method. Methods. 2001;25:402-8

24. Diatchenko L, Lau F, Campbell AP, Chenchik A, Mogadam F, Huang B, et al. Suppression subtractive hybridization: a method for generating differentially regulated or tissue-specific cDNA probes and libraries. PNAS USA. 1996;93:6025-30.

25. Thailayil J, Magnusson K, Godfray HC, Crisanti A, Catteruccia F. Spermless males elicit large-scale female responses to mating in the malaria mosquito Anopheles gambiae. PNAS USA. 2011;108:13677-81.

26. Avila FW, Sirot LK, LaFlamme BA, Rubinstein CD, Wolfner MF. Insect seminal fluid proteins: identification and function. Annual Rev Entomol. 2011;56:21-40.

27. Timmons L, Court DL, Fire A. Ingestion of bacterially expressed dsRNAs can produce specific and potent genetic interference in Caenorhabditis elegans. Gene. 2001;263:103-12.

28. Burtis KC. The regulation of sex determination and sexually dimorphic differentiation in Drosophila. Curr Opin Cell Biol. 1993;5:1006-14.

29. Salvemini M, Mauro U, Lombardo F, Milano A, Zazzaro V, Arca B, et al. Genomic organization and splicing evolution of the doublesex gene, a Drosophila regulator of sexual differentiation, in the dengue and yellow fever mosquito Aedes aegypti. BMC Evol Biol. 2011;11:41.

30. Suckling DM, Stringer LD, Stephens AE, Woods B, Williams DG, Baker G, et al. From integrated pest management to integrated pest eradication: technologies and future needs. Pest Manag Sci. 2014;70:172-89.

31. Price DR, Gatehouse JA. RNAi mediated crop protection against insects. Trends Biotechnol. 2008:26:393-400.

32. Huvenne H, Smagghe G. Mechanisms of dsRNA uptake in insects and potential of RNAi for pest control: a review. J Insect Physiol. 2010;56:227-35.

33. Yu N, Christiaens O, Liu J, Niu J, Cappelle K, Caccia S, et al. Delivery of dsRNA for RNAi in insects: an overview and future directions. Insect Sci. 2013;20:4-14.

34. Salvemini M, D'Amato R, Petrella V, Aceto S, Nimmo D, Neira M, et al. The orthologue of the fruitfly sex behaviour gene fruitless in the mosquito Aedes aegypti: evolution of genomic organisation and alternative splicing PLoS One. 2013:8:e48554

35. Scali C, Catteruccia F, Li Q, Crisanti A. Identification of sex-specific transcripts of the Anopheles gambiae doublesex gene. J Exp Biol. 2005;208:3701-9.

36. Bellini R, Calvitti M, Medici A, Carrieri M, Celli G, Maini S. Use of the sterile insect technique against Aedes albopictus in Italy: First Results of a pilot trial. In: Vreysen MJB, Robinson AS, Hendrichs J, editors. Area-Wide Control of Insect Pests. Dordrecht: Springer; 2007. p. 505-15.

37. Lance DR, McInnis DO. Biological basis of the sterile insect technique. In: Dyck VA, Hendrichs J, Robinson AS, editors. Sterile Insect Technique: Principles and Practice in Area-Wide Integrated Pest Management. Dordrecht: Springer; 2005. p. 70-94.

38. Ito $Y$, Yamamura K. Role of population and behavioural ecology in the sterile insect technique. In: Dyck VA, Hendrichs J, Robinson AS, editors. Sterile Insect Technique: Principles and Practice in Area-Wide Integrated Pest Management. Dordrecht: Springer; 2005. p. 178-208.

39. Hunter WB, Glick E, Paldi N, Bextine BR. Advances in RNA interference: dsRNA treatment in tree and grapevines for insect pest suppression. Southwest Entomol. 2012;37:85-7.

40. Posiri P, Ongvarrasopone C, Panyim S. A simple one-step method for producing dsRNA from E. coli to inhibit shrimp virus replication. J Virol Methods. 2013:188:64-9.

41. Kumar A, Wang S, Ou R, Samrakandi M, Beerntsen BT, Sayre RT. Development of an RNAi based microalgal larvicide to control mosquitoes MalariaWorld J. 2013:4:1-7.

42. Baum JA, Bogaert T, Clinton W, Heck GR, Feldmann P, Ilgan O, et al. Control of coleopteran insect pests through RNA interference. Nature Biotech. 2007:25:1322-6.

43. Whyard S, Singh AD, Wong S. Ingested double-stranded RNAs can act as species-specific insecticides. Insect Biochem Mol Biol. 2009;39:824-32. 